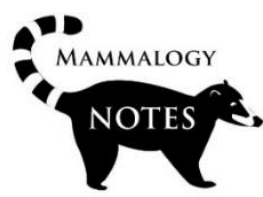

\title{
Punzones de hueso de Odocoileus virginianus (Artiodactyla: Cervidae) de un depósito arqueológico del Posclásico temprano en la costa de Jalisco, México
}

\author{
Fabio G. Cupul-Magaña1*(i), Joseph B. Mountjoy \\ 1 Centro Universitario de la Costa, Universidad de Guadalajara, Av. Universidad 203, Delegación Ixtapa, C. P. 48280, Puerto \\ Vallarta, Jalisco, México. \\ * Correspondencia: fabiocupul@gmail.com
}

\section{Resumen}

Se describen punzones elaborados con huesos metapodiales de venado cola blanca (Odocoileus virginianus) encontrados en el sitio arqueológico Arroyo Piedras Azules (1215 de nuestra era) del centro occidente de México. Se especula que fueron utilizados para fabricación de vestimentas, uso en telares o perforaciones corporales.

Palabras clave: Arroyo Piedras Azules, Aztatlán, huesos metapodiales.

Abstract

Punches made with metapodial bones of white-tailed deer (Odocoileus virginianus) found at the Arroyo Piedras Azules archaeological site (1215 AD) in western central Mexico are described. It is speculated that they were used for clothing manufacturing, use on looms, or body piercing.

Key words: Arroyo Piedras Azules, Aztatlán, metapodial bones.

El sitio Arroyo Piedras Azules es un depósito arqueológico de aproximadamente 3 ha de superficie localizado en la costa norte del estado de Jalisco, México (20,26185, -105,57505 WGS84); elevación 29 msnm (Figura 1a). Aunque el sitio tiene evidencia de habitación durante el Preclásico medio y el Preclásico tardío/Clásico temprano, así como el Posclásico tardío, el enfoque de las investigaciones fue durante su habitación en el Posclásico temprano de la cultura arqueológica Aztatlán, que empezó en el $1215 \pm 30$ de nuestra era (años calibrados al 95\% de certeza, Cupul-Magaña \& Mountjoy, 2018; Mountjoy et al. 2020).

Las excavaciones en el sitio se realizaron en los años 2015, 2017 y 2018, y estuvieron enfocadas en una capa de aproximadamente $1 \mathrm{~m}$ de grueso formada de desechos domésticos de la habitación Aztatlán. De esa capa se recuperaron 14.693 fragmentos de cerámica Aztatlán, figuras antropomorfas de cerámica, herramientas de piedra, objetos de 
cobre, joyería de concha, además de abundantes restos de la comida cotidiana como conchas de bivalvos y caracoles, huesos de reptiles, peces, aves y mamíferos, así como elotes carbonizados (Mounyjoy et al. 2020).

Se cree que la presencia de huesos animales en el depósito probablemente evidencia su uso como recurso alimenticio u ornamental (Cupul-Magaña et al. 2016, 2018; Cupul-Magaña \& Mountjoy, 2017, 2018). Entre los huesos de mamíferos encontrados están los de perro Canis lupus familiaris Linnaeus, 1758 (Carnivora: Canidae), conejo mexicano de monte Sylvilagus cunicularius, Waterhouse, 1848 (Lagomorpha: Leporidae) y venado cola blanca Odocoileus virginianus (Zimmermann, 1780) (Cupul-Magaña \& Mountjoy 2018).

Para el venado cola blanca, se han hallado dientes, mandíbulas y fragmentos de cornamentas (Cupul-Magaña \& Mountjoy 2018). Sin embargo, no fue sino hasta la revisión reciente del material arqueológico obtenido de tres pozos de prueba (de $1 \mathrm{~m}^{2}$ y $190 \mathrm{~cm}$ de profundidad) de las campañas de exploración de 2017 y 2018, que se encontraron tres fragmentos de punzones fabricados con hueso de venado. Así, el material presentado en esta nota proviene de las excavaciones del pozo \#3 (capa de 130-180 cm de profundidad; 8 de marzo de 2017), pozo \#11 (capa de 90-100 cm de profundidad, 17 de julio de 2017) y pozo \# 20 (capa 50-70 cm de profundidad; 23 de abril de 2018).

Los fragmentos encontrados son extremos de punzones fabricados con huesos metapodiales de O. virginianus: uno del mango (Figuras $1 \mathrm{~b}-\mathrm{d}$ ) y dos de la punta (Figuras 1g-j). El mango es de tono pálido y las puntas son de color marrón rojizo semioscuro por efecto de las quemaduras del fuego a distintas temperaturas (Beisaw 2013). Así, su coloración y forma indican que probablemente sean fragmentos de diferentes punzones. La identificación taxonómica del material óseo se realizó con el apoyo de los trabajos de Olsen (1964), Schaller (1992) y Adams \& Crabtree (2012), así como por comparación directa con material de referencia (Figuras 1e-f) depositado en la Colección de la Estación de Biología Chamela del Instituto de Biología de la Universidad Nacional Autónoma de México. En esta misma colección se depositó el material (sin números de identificación) presentado en esta nota.

El mango es un hueso recto de $5 \mathrm{~cm}$ de largo $(2 \mathrm{~cm}$ de ancho en su parte proximal, $1,5 \mathrm{~cm}$ de ancho en su parte distal y grosor medio de $0,5 \mathrm{~cm}$ ), con el margen proximal recto y ligeramente curvo hacia los extremos, el margen distal roto, y los márgenes laterales redondeados que tienden a converger de la parte proximal a la distal del hueso (Figura 1b). La superficie externa es ligeramente cóncava y la interna esta marginada y con un surco longitudinal que reduce su anchura de la parte proximal a la distal (Figura 1d). Las superficies y los márgenes tienen una apariencia pulida y son suaves al tacto, resultado del trabajo humano realizado en su elaboración o uso (Beisaw 2013). Además de las características anteriores, en el margen derecho de la superficie externa del mango (Figura 1c), se destaca una concavidad o muesca estrecha y alargada de $3 \mathrm{~cm}$ de largo que corresponde a la sutura anterior del metatarso (no presente en el metacarpo; asimismo, por la posición de la sutura, se trata del metatarso derecho) de un venado cola blanca (Figuras 1c-d). Este detalle evidencia que en la fabricación del punzón se utilizó el eje mayor del hueso del metatarso. También, se presentan marcas de cortes entre la parte media a la apical de la superficie externa del mango (Figura 1b), relacionadas tal vez con el desollamiento, la extracción de carne o de tendones del hueso del animal (Beisaw 2013; Montero López 2014). 
En cuanto a las puntas, estas miden 3,5 y $3 \mathrm{~cm}$ de longitud, respectivamente. La región proximal está rota y la distal ligeramente redondeada (rota en una de ellas). Las superficies externa e interna se observan y perciben como lo descrito para el mango. Los márgenes laterales tienden a converger, desde la región apical a la distal, hasta formar una punta roma. Por la forma de sus superficies y por corresponder a la parte distal del punzón, se fabricaron con huesos metapodiales de venado cola blanca, más no tenemos evidencia para afirmar si se trata de huesos del metatarso o metacarpo (Figuras 1e-f).
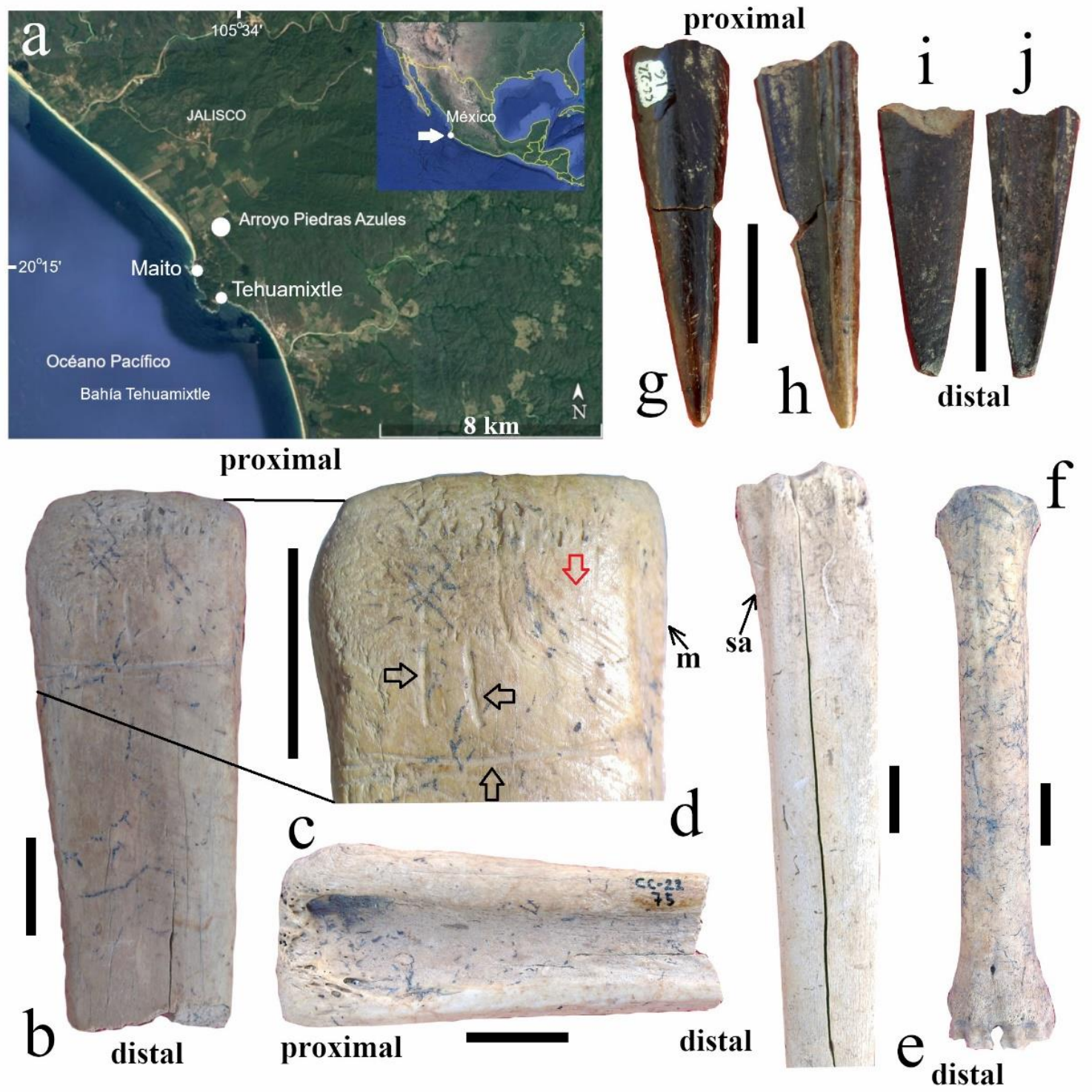

FIGURA 1. a) Localización del sitio Arqueológico Arroyo Piedras Azules en el occidente de México. bj) Huesos de venado cola blanca. Mango del punzón (pozo \# 11): b) superficie externa, c) interna y d) detalle de la región proximal con marcas de corte (flechas negras) y abrasión (fecha roja); $m$ = muesca. e) Vista lateral anterior de metatarso izquierdo de la colección de referencia; sa = sutura anterior. f) Vista dorsal de metacarpo derecho de la colección de referencia. Punta de punzones: superficie interna y externa de muestra del pozo \# 3 ( y y h) y pozo \# 20 (i e j), respectivamente. Marcas de escala de $1 \mathrm{~cm}$. 
Para otras regiones del occidente de México, se ha documentado que el venado cola blanca fue empleado como un excelente proveedor de carne y materia prima, piel o huesos, que pudieron ser aprovechados para la elaboración de punzones y agujas utilizados en la confección de vestimentas (Rodríguez Galicia et al. 2012). También, es posible que los punzones se utilizaran para telar (Mathiowetz 2020), pues esa función produce un buen brillo de pulimiento en sus orillas (como el observado en las muestras) (Mountjoy et al. 2020); así como para perforar los lóbulos de las orejas para usar adornos, tal como se observa en la iconografía de la cerámica encontrada en el sitio arqueológico (Mountjoy et al. 2020).

\section{AGRADECIMIENTOS}

Al Centro del Instituto Nacional de Antropología e Historia (INAH) en Jalisco, por su permiso para excavar la zona (número 401.15.1-2017/34). A Rafael García de Quevedo Machain por su apoyo con los datos de recolecta y excavación de los pozos. Al editor asociado y a los revisores anónimos por sus valiosos comentarios.

\section{REFERENCIAS}

Adams B, Crabtree P. 2012. Comparative osteology: A laboratory and field guide of common North America animals. Academic Press, Amsterdam, Paises Bajos.

Beisaw AM. 2013. Identifying and interpreting animal bones: A manual. Texas A \& M University Press, College Station.

Cupul-Magaña FG, Mountjoy JB. 2017. Restos de la pardela patas rosadas Ardenna creatopus (Procellariiformes: Procellariidae) en el sitio arqueológico Arroyo Piedras Azules, Jalisco, México. Ciencia y Mar. 21(63): 19-23.

Cupul-Magaña FG, Mountjoy JB. 2018. Huesos de mamíferos (Carnivora: Canidae, Artiodactyla: Cervidae y Lagomorpha: Leporidae) en un depósito cultural de la costa de Jalisco, México. Mammology Notes. 4(2): 15-17.

Cupul-Magaña FG, Mountjoy JB, Escobedo-Galván AH. 2016. Reptiles in a cultural deposit in western Mexico. Mesoamerican Herpetology. 3(3): 808-810.

Cupul-Magaña FG, Mountjoy JB, García de Quevedo Machain R. 2018. Restos óseos de pez loro (Scarus perrico) y jurel (Caranx canius) en un depósito cultural Aztatlán en Maito, Jalisco, México. Acta Pesquera. 4(8): 58-61.

Mathiowetz M. 2020. Weaving our life: The economy and ideology of cotton in Postclassic West Mexico, in: Ancient West Mexicos: Time, space, and diversity. University Press of Florida, Gainsville. p. 302-348.

Montero López C. 2014. Infiriendo el contexto arqueológico a través de la tafonomía: el uso del venado cola blanca (Odocoileus virginianus) en Chinikihá, Chiapas, in: La arqueología de los animales en Mesoamérica. Lockwood Press, Atlanta, GA. p. 339-375.

Mountjoy JB, Cupul-Magaña FG, García de Quevedo-Machain R, López Mestas Camberos ML. 2020. The Early Postclassic Aztatlán colonization of the Pacific coast of Jalisco. In: Ancient West Mexicos: Time, space, and diversity. University Press of Florida, Gainsville. p. 131-156.

Olsen SJ. 1964. Mammal remains from archaeological site. Part1. Southeastern and southwestern United States. Papers of the Peobody Museum of Archaeology and Ethnology. 56(1): 1-162. 
Rodríguez Galicia B, Valadez Azúa R, Cabrero García MT, García Giménez GC. 2012. Arqueofauna del sitio El Piñón, cultura Bolaños, Jalisco, México. Revista del Museo de Antropología. 5: 203212.

Schaller, O., 1992. Illustrated veterinary anatomical nomenclature. Enke Verlag, Stuttgart, Alemania.

Editor: Diego J. Lizcano Recibido 2020-05-15

Revisado 2020-05-20

Aceptado 2020-05-29

Publicado 2020-06-27 